\title{
Elección y Preferencia del Parto Domiciliario en Callao, Perú
}

Carolina Tarqui-Mamani ${ }^{1}$ y Alejandro Barreda-Gallegos ${ }^{2}$

${ }^{1}$ Licenciada en Obstetricia. Instituto Nacional de Salud, Lima Perú. E-mail: carobtm@hotmail.com, ctarqui@ins.gob.pe.

${ }^{2}$ Médico. Facultad de Medicina, Universidad Nacional Mayor de San Marcos, Lima, Perú. E-mail: abarreda@unmsm.edu.pe.

Recibido: 19 Enero 2006/Enviado para Modificación: 8 Abril 2006/Aceptado 14 Septiembre 2006

\section{RESUMEN}

Objetivo Identificar los factores personales y de la atención prenatal relacionados con la elección y preferencia domiciliaria del lugar del parto.

Material y métodos El estudio incluyó a 52 casos: mujeres en edad fértil (MEF) cuyo último parto fue domiciliario; y 208 controles: MEF con parto institucional residentes en la misma área geográfica. El estudio se realizó en la Micro red Pachacútec (Callao-Perú). El análisis fue bivariado y multivariado.

Resultados La edad promedio de los casos fue 28 años (DS=6,5), y de los controles fue 26 años (DS=6,1). El análisis bivariado demostró la asociación significativa de diversos factores pero al aplicar el análisis multivariado éstos no fueron confirmados. Finalmente, los factores de riesgo fueron: el domicilio como lugar previsto de parto $w=34,8(p=0,0) ; O R=31,7 \quad(I C=10,1-99,8)$ y el proveedor de la atención prenatal $w=7,0(p=0,0)$; OR=0,1 (IC=0,0-0,5).

Conclusiones El domicilio como lugar previsto de parto y el proveedor de la atención prenatal son los factores que están altamente asociados con el parto domiciliario.

Palabras Clave: Parto domiciliario, estudio de casos y controles, factores de riesgo, continuidad de la atención del paciente, salud materna, barreras de comunicación, Perú (fuente: DeCS, BIREME).

\section{ABSTRACT \\ Choice of and preference for home child-birth in Callao, Perú}

Objective Identifying the personal factors and prenatal attention associated with choosing the home for giving birth.

Material and Methods The study included 52 cases (fertile women whose last childbirth had been at home) and 208 controls (fertile women residing in the same geographical area). The study was carried out in the Pachacútec micro- 
network. Analysis was both bivariate and multivariate.

Results The cases' average age was 28 (6,5 SD) and 26 for controls (6,1 SD). Bivariate analysis demonstrated a significant association for different factors but these they were not confirmed when multivariate analysis was applied. The risk factors were the home as foreseen place for childbirth $w=34,8(p=0,0)$; $\mathrm{OR}=31,7(\mathrm{Cl}=10,1 ; 99,8)$ and prenatal attention supplier $w=7,0(p=0,0) ; O R=0,1$ $(\mathrm{Cl}=0,0 ; 0,5)$.

Conclusions The home as foreseen place for childbirth and prenatal attention supplier were the factors which were highly associated with home childbirth.

Key Words: Home childbirth, case-control study, risk-factor, patient care continuity, communication, barriers, Perú (SOURCE: MESH, NLM).

$\mathbf{U}$ n problema importante de salud pública es el alto índice de mortalidad materna (1). Por tal razón, el Ministerio de Salud del Perú tiene como prioridad disminuir los altos índices de mortalidad materna y perinatalpara lo cual se propuso diversas estrategias de Intervención contenidas en el Plan Nacional para la Reducción de Mortalidad Materna (2). Estas estrategias han estado orientadas a mejorar las coberturas de los servicios de salud, promoviendo el control prenatal y el parto institucional, el cual tiene como objetivo garantizar un parto seguro que no ponga en riesgo la vida de la gestante (3). Sin embargo, persisten los reportes discordantes entre las coberturas de la atención del control prenatal (77,2 \%) y la atención del parto institucional (49,5 \%) (4). Entre los factores que incrementan la preferencia del parto domiciliario tenemos: 1. Personales (la edad, el bajo nivel educativo, el estado conyugal, el embarazo no deseado, el antecedente de parto domiciliario, y la procedencia rural). 2. Factores de la atención prenatal (el inicio tardío del control prenatal, el inadecuado número de controles prenatales, la puntualidad en la atención recibida, la falta de visita domiciliaria, la poca capacidad resolutiva del establecimiento de salud y el tipo de personal de salud que tiene a su cargo la atención prenatal). El objetivo fue identificar los factores personales y factores de la atención prenatal relacionados con la elección y preferencia domiciliaria del lugar del parto. La hipótesis: existen factores de riesgos personales y factores de la atención prenatal que están relacionados con la elección y preferencia domiciliaria del lugar del parto. Hasta el momento se han difundido investigaciones acerca de la ocurrencia de partos domiciliarios; sin embargo, ninguno de éstos, permiten establecer correlaciones entre variables y establecer grados de asociación estadísticamente significativos entre los diferentes factores y el lugar del parto. 


\section{MATERIALES Y MÉTODOS}

Se realizó un estudio observacional, analítico, de casos y controles, en 260 mujeres en edad fértil (MEF) con antecedente de parto, en la jurisdicción de la Micro red Pachacútec. El estudio fue revisado y aprobado por el Comité de Investigación de la Dirección de Salud Callao y por el Comité de Ética de la Facultad de Medicina de la Universidad Nacional Mayor de San Marcos. Todos los participantes firmaron el consentimiento informado para ser incluidos en el estudio.

Se definió como caso a las mujeres en edad fértil cuyo último parto fue domiciliario, entre los años 2000 y 2001, y los controles a las mujeres en edad fértil residentes en la misma área geográfica de cada caso con antecedente de parto institucional en el mismo periodo.

Todas las participantes del estudio tuvieron entre 15 y 19 años, residían en la jurisdicción de la Micro red Pachacútec, la atención del parto fue vía vaginal, sólo se incluyeron los casos de cesárea no programados durante el embarazo y que expresaron su deseo de participar del estudio previa explicación del equipo investigador.

No existe un marco muestral real de los sujetos de estudio dado el problema de sub registro existente en el Sistema de Salud. Por tal razón, el marco muestral corresponde al plano de manzanas, el cual se basa en los mapas que conforman la Micro red Pachacútec. El tamaño muestral se obtuvo a través del programa EPI INFO (5), para lo cual se tomó como referencia el factor menos frecuente (antecedente de parto domiciliario). Se fijó: margen de error de $5 \%$, nivel de confianza del $95 \%$ y potencia de $80 \%$. Según el cálculo obtenido el tamaño de muestra requerido fue $260 \mathrm{MEF}$. La relación caso:control fue 1:4. Los casos fueron 52 MEF y el grupo control 208 MEF. Se realizó una búsqueda poblacional de los casos, para lo cual se aplicó el muestreo sistemático circular con arranque aleatorio. La selección de los controles, se realizó luego de la identificación de cada caso en la población, la cual consistió en asignar al azar 4 controles que viven en la misma zona de residencia del caso identificado.

El presente estudio utilizó un cuestionario para la recolección de datos y se aplicó a todas las MEF incluidas en el estudio, luego se realizó la verificación de algunos datos con la historia clínica respectiva. El cuestionario tuvo tres secciones con preguntas sobre factores personales, aspectos relacionados al 
último parto y factores relacionados con la atención prenatal. En la mayoría de los casos se asumió definiciones operacionales convencionales con valores "si" o "no". El instrumento empleado se construyó utilizando como base la Encuesta Nacional de Demografía y la Salud Familiar (6) y la encuesta sobre la evaluación del control prenatal en el hospital Kennedy (7). Finalmente, antes de ser aplicado, se realizó un estudio preliminar en MEF residentes en el asentamiento humano Mi Perú.

Se utilizó un análisis bivariado de cada una de las variables explicativas y el lugar de parto. Las pruebas estadísticas utilizadas para establecer diferencias y asociaciones fueron el Chi cuadrado con un nivel de significación de $\mathrm{p}<0,05$ para un nivel de confianza del 95 \%. Luego, se hizo cálculos de Odds Ratio. La segunda etapa consistió en aplicar la regresión logística en cada dimensión identificada. Finalmente, se realizó una regresión logística global, en la cual solo ingresaron las variables estadísticamente significativas.

\section{RESULTADOS}

Descripción de los casos y controles

El 60 \% de la población de estudio procede de la sierra, 25 \% de la selva y el resto de los alrededores de Lima. La edad promedio en el grupo de casos fue de 28 años (DS=6,5) y la edad promedio en los controles fue de 26 años (DS=6,1).

En la primera etapa se realizó el análisis bivariado y se encontró que las variables asociadas con la preferencia de atención domiciliaria del parto fueron: la procedencia rural: $\mathrm{x}^{2}=26,5$; $\mathrm{OR}=10,5$ (IC:3,4-35,9), el bajo nivel educativo $x^{2}=30,7(p=0,0)$, el antecedente de hijo muerto $x^{2}=13,5 ; O R=4,7$ (IC:1,8-13,0), el antecedente de partos domiciliarios $x^{2}=168,5$; OR=1020,4 (IC: 134,3-7480), el embarazo no deseado $x^{2}=12,9$; OR=3,3 (IC:1,6-6,9), el domicilio como lugar previsto de parto $x^{2}=88,1 ; \mathrm{OR}=26,3$ (IC:10,7-67,0), el no tener atención prenatal $\mathrm{x}^{2}=38,2 ; \mathrm{OR}=8,7$ (IC: 3,8-20,3), el lugar en el que se realizó el control prenatal $\mathrm{x}^{2}=18,3(\mathrm{p}=0,0)$, el proveedor de la atención prenatal $\mathrm{x}^{2}=29,9(\mathrm{p}=0,0)$. En la segunda etapa se aplicó la regresión logística en las dimensiones identificadas, y se encontró que las únicas variables asociadas significativamente fueron: el domicilio como lugar previsto de parto: $\mathrm{w}=7,0(\mathrm{p}=0,0) ; \mathrm{OR}=5,8 \mathrm{IC}=1,6-21,0)$ y el tipo de profesional que tuvo a su cargo la atención del control prenatal $\mathrm{w}=12,6$ $(\mathrm{p}=0,0) ; \mathrm{OR}=0,0(\mathrm{IC}=0,0-0,2)$, este último se comportó como factor protector. Finalmente, se realizó un último análisis de regresión logística, en el cual solo

ingresaron las variables significativas en el análisis previo y solo resultaron 
realmente asociados con la preferencia del parto domiciliario: el domicilio como lugar previsto de parto $\mathrm{w}=34,8(\mathrm{p}=0,0) ; \mathrm{OR}=31,7(\mathrm{IC}=10,1-99,8)$ y el tipo de profesional que tuvo a su cargo la atención prenatal $\mathrm{w}=7,0(\mathrm{p}=0,0)$; $\mathrm{OR}=0,1$ $(\mathrm{IC}=0,0-0,5)$.

\section{DISCUSIÓN}

La ocurrencia de partos domiciliarios se relaciona con la decisión de buscar o no atención médica, sin embargo, también guarda relación con la confianza que tienen las mujeres con el proveedor de salud (1), lo cual se basa en muchos factores como el horario del servicio, tiempo de viaje, tiempo de espera, disponibilidad de médicos o tratamientos y trato personal que reciben los pacientes10, también es apropiado decir que el parto domiciliario guarda relación con la confianza que tienen las mujeres con el proveedor de salud y la planeación del embarazo (intención, aceptación y deseo) (8).

De acuerdo con el análisis bivariado, la ausencia de la atención prenatal aumenta en casi 9 veces el riesgo de presentar parto domiciliario comparado con mujeres que si tuvieron atención prenatal, algunos señalan que los factores que mejor predicen el parto institucional son el mayor número de controles prenatales y la calidad técnica de la atención (9).

Llamó la atención que la edad no resultó asociada con la elección domiciliaria, dado que algunos estudios consideran que la edad es un marcador de riesgo y presenta cierta relación con el control prenatal o el parto domiciliario, es así que alguno afirman que a menor edad de la madre la probabilidad del control prenatal y del parto institucional disminuye (9); lo cual se explica por la necesidad de algunas mujeres de tratar de mantener oculto el embarazo (10) ya sea porque fue producto de alguna relación considerada "indebida" o porque se dio en el momento inadecuado, situación frecuente en las adolescentes embarazadas dado que conlleva a la interrupción brusca de sus planes de vida y los serios problemas que se originan en el entorno familiar (11).

Si se toma en cuenta solamente los resultados encontrados en el análisis multivariado final, es posible decir que los principales factores relacionados con la preferencia del parto domiciliario son: el domicilio como lugar previsto de parto y el proveedor de la atención prenatal.

El domicilio como lugar previsto de parto, al parecer se genera como producto 
de experiencias previas positivas y negativas vividas durante el proceso de parto por la gestante, amigas y familiares (12), las mismas que pueden ser recordadas como un evento gratificante o como un suceso terrible y difícil de olvidar. Se cree que las actitudes de apoyo, protección y atención en el momento del parto son valorado por las mujeres y lo recuerdan llenas de satisfacción y alegría, en cambio la falta de apoyo, la soledad, la sensación de abandono, las actitudes discriminativas, el aislamiento de sus seres queridos, el maltrato físico o psicológico, son sucesos que no se olvidan fácilmente, debido a la sensibilidad, susceptibilidad y vulnerabilidad que presenta la mujer como producto del proceso de gestación, situación que se agudiza en el momento del parto. El presente estudio encontró que el riesgo de presentar parto domiciliario es 34 veces mayor en las mujeres cuyo lugar previsto de parto sea el domicilio comparado con mujeres que optaron por la atención institucional. Cabe añadir, que otro aspecto que podría influir en el lugar previsto de parto es la brecha que existe entre los servicios de salud y la población que podría incrementar el rechazo a la atención institucional, al respecto algunos estudios muestran un serio distanciamiento entre los servicios de salud y la población, lo que se traduce en inaceptables indicadores de salud, especialmente maternos (13). Asimismo, plantea como respuesta al problema de la relación intercultural en la demanda de los Servicios de Salud Reproductiva, la evidencia del desconocimiento de parte del personal de salud de las costumbres, tradiciones y consideraciones culturales de la demanda rural potencial, la imposición de procedimientos de atención o normas técnicas orientadas fundamentalmente hacia la obtención de metas institucionales, antes que contribuir a alcanzar condiciones de salud y bienestar, debilidades en la oferta de los servicios de salud como consecuencia del sistema local de salud, y la poca iniciativa local institucional o incorporar dentro de la oferta de las prestaciones, éstos aspectos (13). Según Olivera, es posible disminuir la mortalidad materna mediante la adaptación de los servicios de salud a la realidad cultural de las gestantes, la cual se inicia con una fase de información, adecuación cultural consistente en la atención de trabajo de parto vertical o de rodillas, de este modo se consigue evitar el rechazo al parto institucional, adaptándose el personal de salud a las exigencias culturales de las gestantes; la permanencia de la gestante cerca de un establecimiento de salud.

Existe una gran distancia cultural entre la población y los servicios de salud, dado que la gente acude a ellos sólo cuando reconoce la presencia de una alteración en su salud (11), es así, que la gestante acude por primera vez al establecimiento de salud cuando se aproxima la fecha del parto, cuando inicia el trabajo de parto o cuando siente alguna anormalidad durante el embarazo. Otro 
problema relacionado con el acceso a los servicios, lo constituyen las limitaciones económicas y la afiliación al sistema general de seguridad social de salud (7). Actualmente, el gobierno peruano promueve la atención de la gestante a través del seguro integral de salud, mediante el cual se garantiza la gratuidad de la atención de la gestante, otro sector de la población es atendida por las entidades prestadoras de salud (Essalud y EPS).

Por otro lado, se encontró que el tipo de proveedor de la atención prenatal es un factor protector para la atención institucional, lo cual significa que el $96 \%$ de las gestantes que sean atendidas por profesional altamente calificado y capacitado optarán por la atención del parto institucional comparado con aquellas gestantes cuyo atención prenatal sea dada por personal poco entrenado en el área.

El primer control del embarazo es crucial para determinar el riesgo de la gestante y establecer el tipo de atención necesario para evitar las complicaciones maternas perinatales que podrían finalizar en muerte materna. Asimismo, prescribir en cada atención las intervenciones probadas como beneficiosas sin necesidad de brindarlas en una gran cantidad de controles (14). Además, es necesario evaluar y determinar la capacidad resolutiva y el nivel de complejidad de cada establecimiento de salud, el cual se basa en la infraestructura, el equipamiento del establecimiento y el recurso humano que tienen a su cargo la atención de la gestante. Al respecto, Urrego refiere que la gestación requiere cuidado materno del personal de salud debidamente entrenado de tal manera que se pueda identificar y controlar los riesgos de forma oportuna, así mismo señala que la experticia del profesional está altamente asociada con el nivel de atención especializada del establecimiento de salud (7).

Una de las debilidades el estudio es el probable sesgo de selección que podría haberse presentado al momento de seleccionar a los participantes que conforman el grupo de casos, aún cuando estuvo basado en una búsqueda poblacional, la cual no implica que necesariamente abarque todos los casos existentes. El sesgo de memoria ha sido minimizado dado que se partió del hecho de que el parto es un evento "único", que la madre difícilmente puede olvidar por lo que se realizó mediciones referidas al último embarazo de las madres seleccionadas.

Una de las dificultades que se tuvo durante el desarrollo del presente estudio fue el cruce de información con la historia clínica de la entrevistada planteado inicialmente dado que en algunos casos fue imposible acceder a éste tipo de 
información, dado que se trata de asentamientos humanos con pocos años de creación por lo cual la gran mayoría de la población proviene de la sierra, selva y de los alrededores de Lima, por tal razón; aún cuando al inicio del estudio se propuso contrastar los datos encontrados no siempre se realizó como tal, dado que los establecimientos de salud en donde se realizó la atención prenatal no siempre dependían de la Dirección de salud Callao y no se contaba con la autorización correspondiente para tener acceso a ciertos documentos.

En conclusión: el parto domiciliario aún es un problema que persiste no solo en las provincias del interior del Perú sino también en los asentamientos urbanomarginales de Lima en donde la gente vive en una situación de pobreza y extrema pobreza; en razón a ello, se sugiere promover la atención multidisciplinaria durante la atención prenatal con especial cuidado a aquellos casos de gestantes que teniendo control de su embarazo en algún establecimiento de salud planifican llevar un parto domiciliario. Entonces es necesario diseñar estrategias que acorten la brecha de la interculturalidad entre el prestador de salud y la gestante e incorporar a los promotores de salud y demás recursos de la comunidad como los principales nexos entre el Sistema de Salud y la Comunidad para la captación oportuna de gestantes. Además, el personal de salud deberá trabajar de forma articulada con las diferentes organizaciones de la comunidad (Gobierno local, organizaciones de base, organizaciones no gubernamentales, instituciones locales, agentes comunitarios de salud, pareja y familia de la gestante) para formar una red activa que cuide, promueva la atención prenatal y la atención institucional del parto

\section{REFERENCIAS}

1. Instituto Nacional de Estadística e Informática. Encuesta demográfica y de salud familiar 2000. Lima: Informe Técnico: INEI; Mayo 2001.

2. Carrasco I. Resultados de las intervenciones llevadas a cabo para reducir la Mortalidad Materna en el Perú. Rev. Instituto Materno Perinatal 2000; 8:10-15.

3. Ministerio de Salud, Programa Nacional de Salud Materna y Perinatal. Plan nacional de acción para la reducción de la mortalidad materna y perinatal 1998-2001. Lima: MINSA; 2002.

4. Ministerio de Salud. Evaluación anual del área de la Dirección General de Salud de las Personas. Lima:DGSP/MINSA; 2002.

5. Fleiss J, Levin B, Cho MP. Statistical methods for rates and proportions. 2da ed. New Jersey: Wiley; 1981. p. 44-45.

6. Instituto Nacional de Estadística e Informática. Variables investigadas con las Endes. Lima: Informe técnico: INEI; 2001. p 221. 
7. Urrego Z, Gonzales E, Fernandez R. Evaluación del Control Prenatal en el Hospital Kennedy Primer Nivel E.S.E. Bogotá 1999-2000; Revista de la Federación Panamericana de Asociaciones de Facultades (Escuelas) de Medicina 2001. 1(4):p12. [Internet]. Disponible en: http://www.fepafem.org.ve/ investigaciones/ pdf/control\%20prenat.pdf. Consultado Enero 10, 2004.

8. Chávez M, Casanueva E. Uso de servicios preventivos de salud materno-infantil en un grupo de mujeres mexicanas. Rev Salud Pública (Bogotá) 2005; 7(1): 1625.

9. Vicuña M. Efecto de las intervenciones del Estado en la utilización de servicios de salud maternos en el Perú, durante 1991-2000. Informe técnico. Lima: INEI; 2002. p. 85.

10. Langer A. El embarazo no deseado: impacto sobre la salud y la sociedad en América Latina y el Caribe. Revista Panamericana de la Salud Pública. Marzo 2002; 11 (3):192-204.

11. Molina R, Luengo X, Sandoval J, González E, Castro R, Molina T. Factores de riesgo del embarazo, parto y recién nacido en adolescentes embarazadas. Centro de Medicina Reproductiva y Desarrollo Integral del Adolescente. Revista Sogia 1998; 5 (1):17 [Internet].. Disponible en http:// www.cemera.uchile.cl/sogia/sogia.html. Consultado, Enero 5, 2004.

12. Dierna R, Salazar X, Vargas R, Nacarado P, Vargas C. El Parto de la Vida en Los Andes y Amazonía del Perú. Informe Final. Proyecto 2000. Convenio de Cooperación entre el Ministerio de Salud del Perú y la Agencia de los Estados Unidos para el Desarrollo Internacional (USAID). Lima: Tarea Gráfica Educativa; 2000. p 255.

13. Suárez N, Coasaca A. Influencia de la Interculturalidad en la demanda de los Servicios de Salud Reproductiva. Compendio de Investigaciones Operativas en salud Materno Infantil. Convenio de Cooperación entre el Ministerio de Salud del Perú y la Agencia de los Estados Unidos para el Desarrollo Internacional (USAID). Lima: Tarea Gráfica Educativa; 2000. p 157-209.

14. Villar J, Ba’aqell H, Piaggio H, Lumbiganon P, Belizán JM, Farnot U., et al. La investigación clínica aleatorizada del control prenatal de la Organización Mundial de la Salud para la evaluación de un nuevo modelo de control prenatal. Lancet. 2001; 357: 1551-1564. 